\title{
Parameter estimation of gravitational waves from precessing black hole-neutron star inspirals with higher harmonics
}

\author{
Richard O'Shaughnessy, ${ }^{1,}$ Benjamin Farr, ${ }^{2}$ Evan Ochsner, ${ }^{1}$ Hee-Suk Cho, ${ }^{3}$ V. Raymond, ${ }^{6}$ \\ Chunglee Kim, ${ }^{4,5}$ and Chang-Hwan Lee ${ }^{3}$ \\ ${ }^{1}$ Center for Gravitation and Cosmology, University of Wisconsin-Milwaukee, \\ Milwaukee, Wisconsin 53211, USA \\ ${ }^{2}$ Department of Physics and Astronomy \& Center for Interdisciplinary Exploration and Research in \\ Astrophysics (CIERA), Northwestern University, Evanston, Illinois 60208, USA \\ ${ }^{3}$ Department of Physics, Pusan National University, Busan 609-735, Korea \\ ${ }^{4}$ Astronomy Program, Department of Physics and Astronomy, Seoul National University, \\ 1 Gwanak-ro, Gwanak-gu, Seoul 151-742, Korea \\ ${ }^{5}$ Korea Institute of Science and Technology Information, Daejeon 305-806, Korea \\ ${ }^{6}$ LIGO Laboratory, California Institute of Technology, MC 100-36, Pasadena California 91125, USA
}

(Received 3 March 2014; published 27 May 2014)

Precessing black hole-neutron star (BH-NS) binaries produce a rich gravitational wave signal, encoding the binary's nature and inspiral kinematics. Using the lalinference_mcmc Markov chain Monte Carlo parameter estimation code, we use two fiducial examples to illustrate how the geometry and kinematics are encoded into the modulated gravitational wave signal, using coordinates well adapted to precession. Extending previous work, we demonstrate that the performance of detailed parameter estimation studies can often be estimated by "effective" studies: comparisons of a prototype signal with its nearest neighbors, adopting a fixed sky location and idealized two-detector network. Using a concrete example, we show that higher harmonics provide nonzero but small local improvement when estimating the parameters of precessing $\mathrm{BH}-\mathrm{NS}$ binaries. We also show that higher harmonics can improve parameter estimation accuracy for precessing binaries by breaking leading-order discrete symmetries and thus ruling out approximately degenerate source orientations. Our work illustrates quantities gravitational wave measurements can provide, such as the orientation of a precessing short gamma ray burst progenitor relative to the line of sight. More broadly, "effective" estimates may provide a simple way to estimate trends in the performance of parameter estimation for generic precessing BH-NS binaries in next-generation detectors. For example, our results suggest that the orbital chirp rate, precession rate, and precession geometry are roughly independent observables, defining natural variables to organize correlations in the highdimensional BH-NS binary parameter space.

DOI: $10.1103 /$ PhysRevD.89.102005

PACS numbers: 04.80.Nn, 95.55.Ym

\section{INTRODUCTION}

Ground based gravitational wave detector networks (notably LIGO [1] and Virgo [2]) are sensitive to the relatively well-understood signal from the lowest-mass compact binaries $M=m_{1}+m_{2} \leq 16 M_{\odot}$ [3-14]. Strong signals permit high-precision constraints on binary parameters, particularly when the binary precesses. Precession arises only from spin-orbit misalignment, occurs on a distinctive time scale between the inspiral and orbit, and produces distinctive polarization and phase modulations [15-17]. As a result, the complicated gravitational wave signal from precessing binaries is unusually rich, allowing high-precision constraints on multiple parameters, notably the (misaligned) spin $[18,19]$. Measurements of the spin orientations alone could provide insight into processes that misalign spins and orbits, such as supernova kicks [20,21],

*oshaughn@gravity.phys.uwm.edu or realign them, such as tides and post-Newtonian resonances [22]. More broadly, gravitational waves constrain the premerger orbital plane and total angular momentum direction, both of which may correlate with the presence, beaming, and light curve [23-25] of any postmerger ultrarelativistic blast wave (e.g., short gamma ray burst) [26]. Moreover, spin-orbit coupling strongly influences orbital decay and hence the overall gravitational wave phase: the accuracy with which most other parameters can be determined is limited by knowledge of black hole $(\mathrm{BH})$ spins [18,27-29]. Precession is known to break this degeneracy [18,19,30-33]. In sum, the rich gravitational waves emitted from a precessing binary allow higherprecision measurements of individual neutron star (NS) masses, black hole masses, and black hole spins, enabling constraints on their distribution across multiple events. In conjunction with electromagnetic measurements, the complexity of a fully precessing gravitational wave signal may enable correlated electromagnetic and gravitational wave 
measurements to much more tightly constrain the central engine of short gamma ray bursts.

Interpreting gravitational wave data requires systematically comparing all possible candidate signals to the data, constructing a Bayesian posterior probability distribution for candidate binary parameters [19,34-41]. Owing to the complexity and multimodality of these posteriors, successful strategies adopt two elements: a well-tested generic algorithm for parameter estimation, such as variants of Markov chain Monte Carlo or nested sampling; and deep insight into the structure of possible gravitational wave signals, to ensure efficient and complete coverage of all possible options $[42,43]$. Owing both to the relatively large number of parameters needed to specify a precessing binary's orbit and to the seemingly complicated evolution, Bayesian parameter estimation methods have only recently been able to efficiently draw inferences about gravitational waves from precessing sources [43]. These improvements mirror and draw upon a greater theoretical appreciation of the surprisingly simple dynamics and gravitational waves from precessing binaries, both in the post-Newtonian limit $[16,17,44-46]$ and strong field [47-52]. For our purposes, these insights have suggested particularly well-adapted coordinates with which to express the dynamics and gravitational waves from precessing BH-NS binaries, enabling more efficient and easily understood calculations. In particular, these coordinates have been previously applied to estimate how well BH-NS parameters can be measured by ground-based detectors [18]. In this work, we will present the first detailed parameter estimation calculations which fully benefit from these insights into precessing dynamics. In short, we will review the natural parameters to describe the gravitational wave signal; demonstrate how well they can be measured, for a handful of selected examples; and interpret our posteriors using simple, easily generalized analytic and geometric arguments.

As a concrete objective, following prior work [18,27] we will explore whether higher harmonics break degeneracies and provide additional information about black holeneutron star binaries. In the absence of precession, higher harmonics are known to break degeneracies and improve sky localization, particularly for LISA [30-32]. That said, these and other studies also suggest that higher harmonics provide relatively little additional information about generic precessing binaries, over and above the leadingorder quadrupole radiation [18,32]. For example, for two fiducial nonprecessing and two fiducial precessing signals, Cho et al. [18], henceforth denoted COOKL, provide concrete predictions for how well detailed parameter estimation strategies should perform, for a specific waveform model. A previous work [27], henceforth denoted OFOCKL, demonstrated that these simple predictions accurately reproduced the results of detailed parameter estimation strategies. In this work, we report on detailed parameter estimation for the two fiducial precessing signals described in COOKL. As with nonprecessing binaries, we find higher harmonics seem to provide significant insight into geometric parameters, in this case the projection of the orbital angular momentum direction on the plane of the sky. As this orientation could conceivably correlate with properties of associated electromagnetic counterparts, higher harmonics may have a nontrivial role in the interpretation of coordinated electromagnetic and gravitational wave observations.

This paper is organized as follows. In Sec. II we describe the gravitational wave signal from precessing BH-NS binaries, emphasizing suitable coordinates for the spins (i.e., defined at $100 \mathrm{~Hz}$, relative to the total angular momentum direction) and the waveform (i.e., exploiting the corotating frame to decompose the signal into three time scales: orbit, precession, and inspiral). Our description of gravitational waves from precessing BH-NS binaries follows Brown et al. [15], henceforth denoted BLO, and [16], henceforth denoted LO. Next, in Sec. III we describe how we created synthetic data consistent with the two fiducial precessing signals described in COOKL in Gaussian noise; reconstructed a best estimate ("posterior distribution") for the possible precessing source parameters consistent with that signal; and compared those predictions with semianalytic estimates. These semianalytic estimates generalize work by COOKL, approximating the full response of a multidetector network with a simpler but more easily understood expression. Using simple analytic arguments, we describe how to reproduce our full numerical and semianalytic results using a simple separation of scales and physics: orbital cycles, precession cycles, and geometry. The success of these arguments can be extrapolated to regimes well outside their limited scope, allowing simple predictions for the performance of precessing parameter estimation. We conclude in Sec. IV.

For the benefit of experts, in the Appendix we discuss the numerical stability and separability of our effective Fisher matrix.

\section{KINEMATICS AND GRAVITATIONAL WAVES FROM PRECESSING BH-NS BINARIES}

\section{A. Kinematics and dynamics of precessing binaries}

The kinematics of precessing binaries are well described in [17], BLO, and LO; see, e.g., Eq. (10) in BLO. In brief, the orbit contracts in the instantaneous orbital plane on a long time scale $1 / \Omega_{\text {rad }}$ over many orbital periods $1 / \Omega_{\phi}$. On an intermediate time scale $1 / \Omega_{\text {prec }}$, due to spin-orbit coupling the angular momenta precess around the total angular momentum direction, which remains nearly constant. On time scales $1 / \Omega_{\text {prec }}$ between $1 / \Omega_{\phi}$ and $1 / \Omega_{\text {rad }}$, the orbital angular momentum traces out a "precession cone." For this reason, we adopt coordinates at $100 \mathrm{~Hz}$ which describe the orientation of all angular momenta relative 


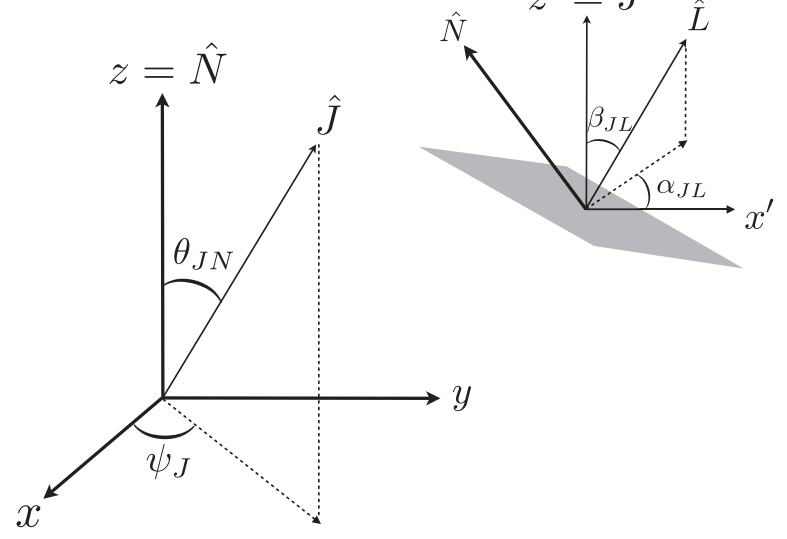

FIG. 1. Coordinate system for the precessing binary. The left coordinate corresponds to the conventional GW radiation frame. $\theta_{J N}\left(\phi_{J N}\right)$ is a polar (azimuthal) angle of the total angular momentum $(J)$ with respect to the radiation vector $(N)$. In the right coordinate $\beta_{J L}\left(\alpha_{J L}\right)$ is a polar (azimuthal) angle of the orbital angular momentum $(L)$ with respect to the total angular momentum $(J)$. In the right coordinate, $N, J$, and $\mathrm{x}^{\prime}$ are coplanar and the shaded region indicates the orbital plane.

to $\vec{J}$; our coordinates are identical to those used in BLO, LO, and COOKL. Relative to a frame with $\hat{z}$ oriented along the line of sight, the total, orbital, and spin angular momenta are described by the vectors ${ }^{1}$ :

$$
\begin{gathered}
\hat{J}=\sin \theta_{J N} \cos \psi_{J} \hat{x}+\sin \theta_{J N} \sin \psi_{J} \hat{y}+\cos \theta_{J N} \hat{z} \\
\hat{L}=\sin \iota \cos \psi_{L} \hat{x}+\sin \iota \sin \psi_{L} \hat{y}+\cos \imath \hat{z}
\end{gathered}
$$$$
\hat{S}_{1}=\sin \theta_{1} \cos \left(\psi_{L}+\phi_{1}\right) \hat{x}+\sin \theta_{1} \sin \left(\psi_{L}+\phi_{1}\right) \hat{y}+\cos \theta_{1} \hat{z}
$$

where in this and subsequent expressions we restrict to a binary with a single spin (i.e., $\vec{S}_{2}=0$ ). Because the orbital angular momentum evolves along a cone, precessing around $\hat{J}$, we prefer to describe the orbital and spin angular momenta in a frame aligned with the total angular momentum $\hat{z}^{\prime}=\hat{J}$ :

$\hat{L}=\sin \beta_{J L} \cos \alpha_{J L} \hat{x}^{\prime}+\sin \beta_{J L} \sin \alpha_{J L} \hat{y}^{\prime}+\cos \beta_{J L} \hat{J}$

where the frame is defined so $\hat{y}^{\prime}$ is perpendicular to $\hat{N}$ as in Fig. 1 [3]:

$$
\hat{y}^{\prime}=-\frac{\hat{N} \times \hat{J}}{|\hat{N} \times \hat{J}|}, \quad \hat{x}^{\prime}=\hat{y}^{\prime} \times \hat{J}=\frac{\hat{N}-\hat{J}(\hat{J} \cdot \hat{N})}{|\hat{N} \times \hat{J}|} .
$$

\footnotetext{
${ }^{1}$ Strictly speaking, the total angular momentum $\vec{J}$ precesses [17]. For the results described in this work, we always adopt the total angular momentum direction evaluated at $f=100 \mathrm{~Hz}$.
}
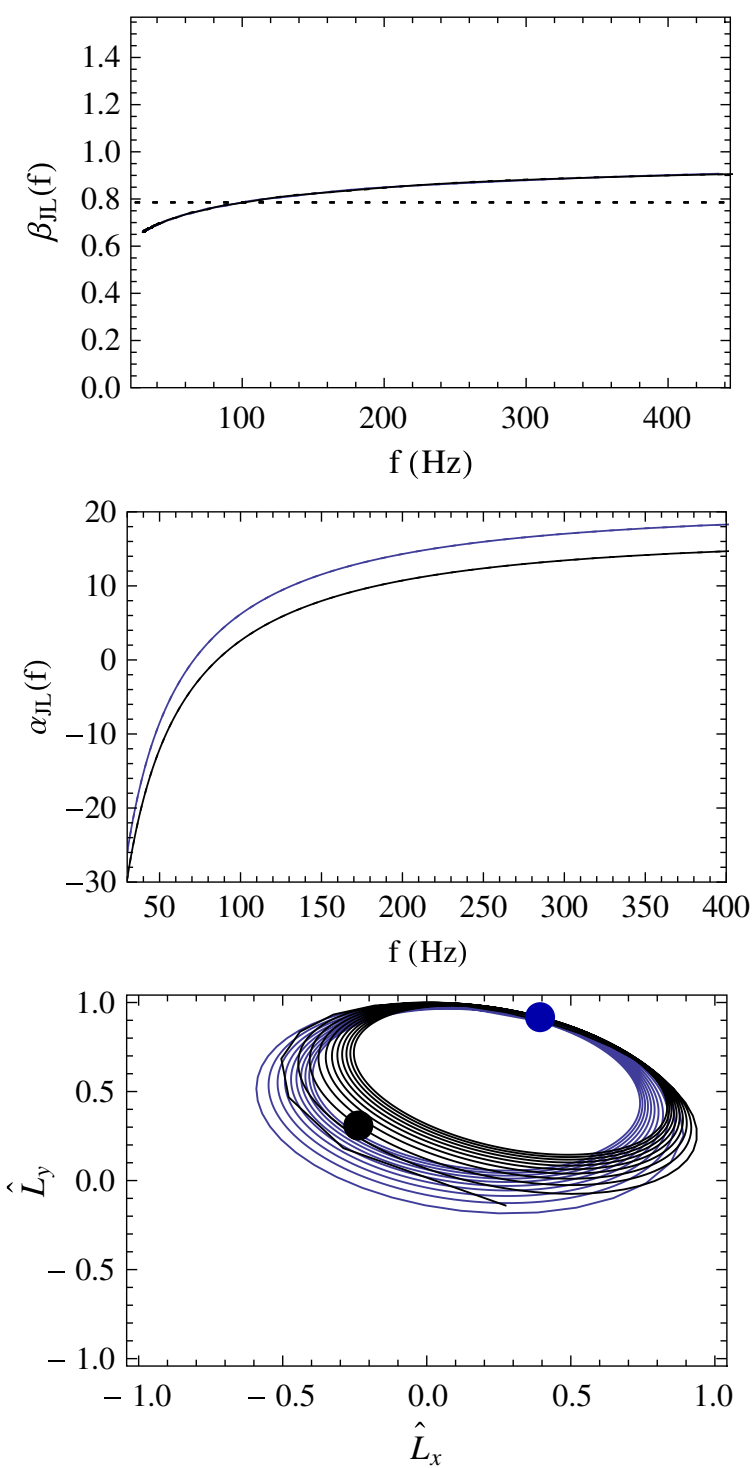

FIG. 2 (color online). Time-dependent geometry. For each of the two fiducial binaries in Table I, a plot of the time-dependent angles $\beta_{J L}(t)$ (top; common to both), $\alpha(t)$ plus an arbitrary integer multiple of $2 \pi$ (center; blue $=\mathrm{A}$; black $=\mathrm{C}$ ), and the two components of the orbital angular momentum direction $\hat{L}$ projected on the plane of the sky (bottom). For completeness, the first two panels also include dotted lines, corresponding to the trajectory adopted when higher harmonics were included and the initial template frequency was reduced. In the bottom panel, solid colored dots indicate the direction of $\hat{L}$ at $100 \mathrm{~Hz}$. The binary precesses roughly 7 times between $30 \mathrm{~Hz}$ and $500 \mathrm{~Hz}$.

In this phase convention for $\alpha_{J L}$, the zero of $\alpha_{J L}$ is one of the two points when $\hat{L}, \hat{J}, \hat{N}$ are all in a common plane, sharing a common direction in the plane of the sky. Transforming between these two representations for $\hat{L}$ is straightforward. For example, given $\hat{N}, \hat{L}$ and $\hat{J}$, we identify $\alpha$ and $\beta_{J L}$ via

$$
\beta_{J L}=\cos ^{-1} \hat{J} \cdot \hat{L}
$$




$$
\alpha_{J L}=\arg \hat{J} \cdot\left[\frac{\hat{L} \times\left(\hat{x}^{\prime}+i \hat{y}^{\prime}\right)}{i \sin \beta_{J L}}\right] .
$$

The spin angular momentum direction is determined from the direction of $\hat{L}$, the direction of $\hat{J}$, and the angle $\theta_{L S}$ between $\hat{S}_{1}$ and $\hat{L}$ :

$$
\begin{aligned}
\hat{S}_{1}= & \sin \left(\beta_{J L}-\theta_{L S}\right) \cos \alpha_{J L} \hat{x}^{\prime}+\sin \left(\beta_{J L}-\theta_{L S}\right) \sin \alpha_{J L} \hat{y}^{\prime} \\
& +\cos \left(\beta_{J L}-\theta_{L S}\right) \hat{J} .
\end{aligned}
$$

Finally, the opening angle $\beta_{J L}$ and the angle $\theta_{L S}$ are related. Using the ratio of $\vec{S}_{1}$ to the Newtonian angular momentum $\vec{L}=\mu \vec{r} \times \vec{v}$ as a parameter,

$$
\gamma(t) \equiv\left|\vec{S}_{1}\right| /|\vec{L}(t)|=\frac{\chi_{1} m_{1}^{2}}{\eta M \sqrt{M r(t)}}=\frac{m_{1} \chi_{1}}{m_{2}} v .
$$

Using this parameter, the opening angle $\beta_{J L}$ of the precession cone (denoted $\lambda_{L}$ in Apostolatos et al. [17]) can be expressed trigonometrically as

$$
\beta_{J L}(t) \equiv \arccos \hat{J} \cdot \hat{L}=\arccos \frac{1+\kappa \gamma}{\sqrt{1+2 \kappa \gamma+\gamma^{2}}}
$$

where $\kappa=\cos \theta_{L S}=\hat{L} \cdot \hat{S}_{1}$. Most BH-NS binaries' angular momenta evolve via simple precession: $\alpha$ increases nearly uniformly on the precession time scale, producing several precession cycles in the band [Eq. (9) in BLO], while $\beta_{J L}$ increases slowly on the inspiral time scale, changing the opening angle only slightly (Fig. 1 in BLO); see Fig. 2.

As described in OFOCKL, we evolve the angular momenta according to expressions derived from general relativity in the post-Newtonian, adiabatic, orbit-averaged limit, an approximation presented in [17] and described in [53]. Though some literature adopts a purely Hamiltonian approach to characterize spin precession [54-59], this orbit-averaged approach is usually adopted when simulating gravitational waves from precessing binaries [6,13,22].

In this work we adopt two fiducial precessing BH-NS binaries, with intrinsic and extrinsic parameters specified in Tables I and II. Figure 2 shows how each binary precesses around the total angular momentum direction $\hat{J}$ and in the plane of the sky. For this mass ratio, the opening angle $\beta_{J L}$ adopted is consistent with randomly oriented $\mathrm{BH}$ spin; see, e.g., Eq. (10) and Fig. 4 of LO. For this sky location, our simplified three-detector gravitational wave network has comparable sensitivity to both linear (or both circular) polarizations.

\section{B. Gravitational waves from precessing binaries}

Precession introduces modulations onto the "carrier signal" produced by the secular decay of the orbit over time. BLO and LO provide a compact summary of the associated signal, in the time and frequency domain. In a frame aligned with the total angular momentum, several harmonics $h_{l m}$ are significant:

$$
h_{+}-i h_{\times}=\sum_{l m} h_{l m} Y_{l m}^{(-2)}
$$

where the harmonics $h_{l m}$ are provided and described in the literature [13]. By "significant," we mean that harmonics have nontrivial power $\rho_{l m}$ :

$$
\rho_{l m}^{2}=2 \int_{-\infty}^{\infty} \frac{\left|\tilde{h}_{l m}\right|^{2}}{S_{h}(f)}
$$

where $S_{h}$ is the fiducial initial LIGO design noise power spectrum. These precession-induced modulations are most easily understood in a corotating frame, as in LO [44,47,49-52,60]:

$$
h_{l m}=\sum_{m^{\prime}} D_{m m^{\prime}}^{l}\left(\alpha_{J L}, \beta_{J L}, \gamma\right) h_{l m^{\prime}}^{\mathrm{ROT}}
$$

where $\gamma=-\int d \alpha \cos \beta_{J L}$ and where $D_{m m^{\prime}}^{l}$ is a Wigner D matrix. In this expression, $h_{l m}^{\mathrm{ROT}}$ is the gravitational wave signal emitted by a binary with instantaneous angular momentum along the $\hat{L}$ axis. In the low-velocity limit, $h_{l m}^{\mathrm{ROT}}$ is dominated by leading-order radiation and hence by equal-magnitude $(l, m)=(2, \pm 2)$ modes. Due to spin-orbit precession with $\beta_{J L} \neq 0$, however, these harmonics are mixed. When $\beta_{J L}$ is greater than tens of degrees, then in the

TABLE I. Fiducial source parameters for precessing binaries. We adopt two fiducial binaries A, C similar to those used in COOKL. All parameters are specified when twice the orbital frequency is $100 \mathrm{~Hz}$. The post-Newtonian signals used in the text terminate at an orbital $f_{\mathrm{MECO}} / 2$, where $f_{\mathrm{MECO}}$ is the smaller of the "minimum energy circular orbits" (hence the acronym) and the frequency at which $\dot{\omega}<0$; the values shown are derived from the same lalsimulation output used in our simulations, estimated from data evaluated at a $32 \mathrm{kHz}$ sampling rate. Comparing with model waveforms that include inspiral, merger, and ringdown, we anticipate this abrupt termination causes relatively little mismatch between our model and the physical signal; see OFOCKL.

\begin{tabular}{lcccccccccccc}
\hline \hline & $m_{1}$ & $m_{2}$ & & & & & & & & & $f_{\text {MECO }}$ \\
Name & $\left(M_{\odot}\right)$ & $\left(M_{\odot}\right)$ & $\chi_{1}$ & $\psi_{J}$ & $\psi_{L}$ & $\imath$ & $\beta_{J L}$ & $\theta_{J N}$ & $\alpha_{J L}$ & $\theta_{L S_{1}}$ & $\phi_{\text {ref }}$ & $(\mathrm{Hz})$ \\
\hline $\mathrm{A}$ & 10 & 1.4 & 1.0 & 1.25 & 0.72 & 1.512 & $\pi / 4$ & 0.730 & 2.95 & 1.176 & 0.93 & 810 \\
$\mathrm{C}$ & 10 & 1.4 & 1.0 & 1.09 & 2.23 & 0.411 & $\pi / 4$ & 0.891 & 5.75 & 1.176 & 1.65 & 810 \\
\hline \hline
\end{tabular}


TABLE II. Source location. Source geocenter event time and sky location. For a sense of scale, this table also provides the time differences between different detector sites, implied by that sky location and event time.

\begin{tabular}{lccccc}
\hline \hline$d$ & $t$ & & & $\Delta t_{L H}$ \\
$(\mathrm{Mpc})$ & $(\mathrm{s})$ & $D E C$ & $R A$ & $\begin{array}{c}\Delta t_{V H} \\
(\mathrm{~ms})\end{array}$ \\
\hline 23.1 & 894383679.0 & 0.5747 & 0.6485 & -3.93 & 5.98 \\
\hline \hline
\end{tabular}

simulation frame all $h_{l m}$ are generally present and significant.

To illustrate that gravitational wave emission from a precessing binary requires several harmonics $h_{l m}$ to describe it when $\beta_{J L}>0$, we evaluate $\rho_{2 m}$, conservatively assuming only the $(2, \pm 2)$ corotating-frame modes are nonzero:

$$
\begin{aligned}
\rho_{2 m}^{2} & \simeq\left[\rho_{2,2}^{\mathrm{ROT}}\right]^{2}\left|d_{2, m}^{2}\left(\beta_{J L}\right)\right|^{2}+\left[\rho_{2,-2}^{\mathrm{ROT}}\right]^{2}\left|d_{-2, m}^{2}\left(\beta_{J L}\right)\right|^{2} \\
& =\rho_{2,2}^{\mathrm{ROT}}\left[\left|d_{2, m}^{2}\left(\beta_{J L}\right)\right|^{2}+\left|d_{-2, m}^{2}\left(\beta_{J L}\right)\right|^{2}\right]
\end{aligned}
$$

where we use orthogonality of the corotating-frame $(2, \pm 2)$ modes. Figure 3 shows that except for a small region $\beta_{J L} \simeq 0$, several harmonics contribute significantly to the amplitude along generic lines of sight, with $\rho_{2 m} / \rho_{22} \gtrsim 0.1$. At this level, these harmonics change the signal significantly, both in overall amplitude $\left(\rho^{2} / 2 * 0.1^{2} \simeq \rho^{2} 0.05\right)$ and in fit to candidate data.

Gravitational waves from precessing BH-NS binaries are modulated in amplitude, phase, and polarization. A generic precessing source oscillates between emitting preferentially right-handed and preferentially left-handed radiation along any line of sight; see [47]. For the scenario adopted here, however, the orbital angular momentum almost always preferentially points towards the observer $(\hat{L} \cdot \hat{N} \gtrsim 0)$, so

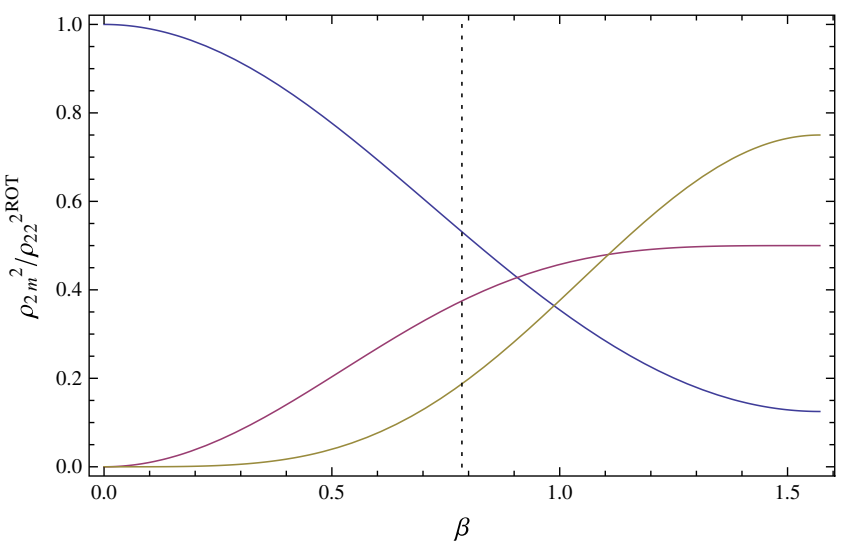

FIG. 3 (color online). Harmonic amplitude versus opening angle. A plot of $\rho_{2 m}^{2} /\left(\rho_{22}^{\text {ROT }}\right)^{2}$ predicted by Eq. (14) for $m=2$ (blue), 1 (red), and 0 (yellow).
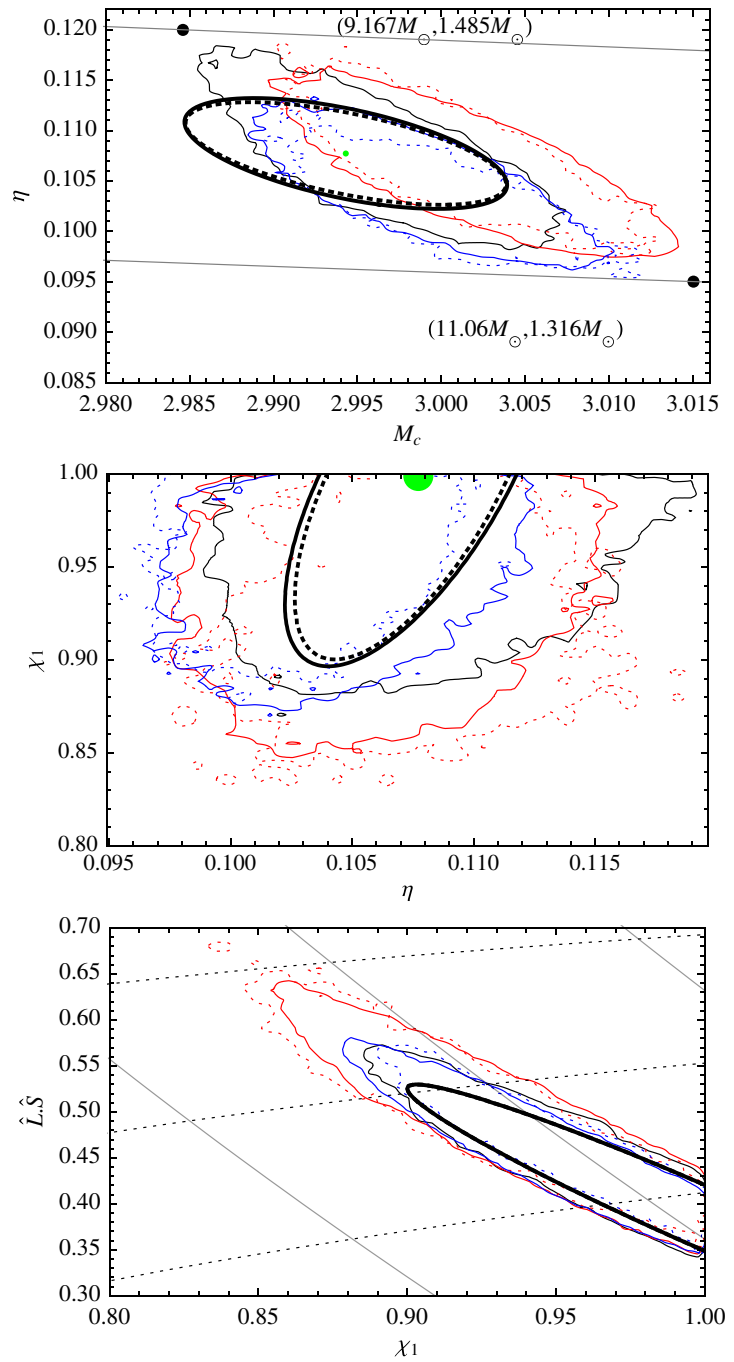

FIG. 4 (color online). Estimating astrophysical parameters (C). For our fiducial binary $\mathrm{C}$, the solid and dotted lines show an estimated $90 \%$ confidence interval with and without higher harmonics, respectively; colors indicate different noise realizations; and the (nearly indistinguishable) thick solid and dashed lines show an approximate effective Fisher matrix result, with and without higher harmonics, not accounting for the constraint imposed by $\chi_{1}<1$. Results for case $\mathrm{A}$ are qualitatively and quantitatively similar. The different panels show different twodimensional projections of the astrophysically relevant parameters of a merging BH-NS binary: the binary mass ratio, black hole spin, and degree of spin-orbit misalignment $\kappa \equiv \hat{L} \cdot \hat{S}_{1}$. Top, center panels: The masses and spin magnitude of the binary can be measured very reliably, consistent with a single Gaussian distribution in four dimensions. The analytic predictions produced by an effective Fisher matrix agree qualitatively but not quantitatively with our simulations. Bottom panel: To guide the eye, the posterior versus $\chi_{1}$ and $\hat{L} \cdot \hat{S}_{1}$ is compared with contours of constant $\beta_{J L}=\cos ^{-1} 0.65,0.7,0.75$ (precession cone opening angle; dotted black) and $\Omega_{p}$ [Eq. (15)] (precession rate; solid black). The precession rate is relatively well constrained by the presence of several $(\simeq 7)$ precession cycles available in data, while the geometry is relatively poorly constrained, relative to the whole $\chi_{1}$ vs $\hat{L} \cdot \hat{S}_{1}$ plane. 
gravitational waves emitted along the line of sight are principally right handed all times.

\section{Symmetry and degeneracy}

Gravitational waves are spin 2: the spin-weight -2 expression $h=h_{+}-i h_{\times}$transforms as $h \rightarrow h \exp (-2 i \psi)$ under a rotation by $\psi$ around the propagation axis. Any gravitational wave signal is unchanged by rotating the binary by $\pi$ around the propagation direction. This exact discrete symmetry ensures that two physically distinct binaries can produce the same gravitational wave signal and can never be distinguished. For this reason, our Markov Chain Monte Carlo (MCMC) evaluate the polarization angle $\psi_{L}$ only over a half-domain $[0, \pi]$ : the remaining halfangle space follows from symmetry. Physically, however, at least two physically distinct spin, $\hat{L}$, and $\hat{J}$ configurations produce the same best-fitting gravitational wave signal. These two spin configurations can be part of the same probability contour or two discrete islands.

For circularly polarized nonprecessing sources, these two spin configurations blur together: the gravitational wave signal is independent of $\psi_{L}-\phi_{\text {orb }}$ (for example), preventing independent measurement of $\psi_{L}$; see, e.g., the top left panel in Fig. 4 of OFOCKL. For generic nonprecessing sources, however, higher harmonics generally isolate the best-fitting gravitational wave signal and thus polarization angles $\psi_{L}$, producing two distinct and exactly degenerate islands of probability over $\psi_{L} \in[0,2 \pi]$; see OFOCKL. For generic precessing sources, the same degeneracy applied to the total angular momentum produces two distinct, exactly degenerate choices for the direction $\psi_{J}$ of $\hat{J}$ in the plane of the sky. This degeneracy cannot be broken. However, at leading order, precessing binaries can still be degenerate in $\psi_{L} \pm \phi_{\text {orb }}$, in the absence of higher harmonics. As we will see below, this degeneracy can be broken, ruling out discrete choices for $\psi_{L}$.

\section{PARAMETER ESTIMATION OF PRECESSING BINARIES}

To construct synthetic data containing a signal, to interpret that signal, and to compare interpretations from different simulations to each other and to theory, we adopt the same methods as used in OFOCKL. Specifically, to determine the shape of each posterior, we employ the lalsimulation and lalinference [19,34] code libraries developed by the LIGO Scientific Collaboration and Virgo Collaboration. As in OFOCKL, we adopt a fiducial threedetector network: initial LIGO and Virgo, with the analytic Gaussian noise power spectrum provided by their Eqs. (1)-(2).

In contrast to the simplified, purely single-spin discussion adopted in Sec. II to describe the kinematics of the physical signal in the data, the model used to interpret the data allows for nonzero, generic spin on both compact objects. That said, because compact object spin scales as the mass squared times the dimensionless spin parameter $\left(S=m^{2} \chi\right)$, in our high-mass-ratio systems the small neutron star's spin has minimal dynamical impact. Our simulations, listed in Table III, show gravitational waves provide almost no information about the neutron star's spin magnitude or direction. For the purposes of simplicity, we will omit further mention of the smaller spin henceforth.

\section{A. Intrinsic parameters}

As shown in Fig. 4, the intrinsic parameters of our relatively loud $(\rho \simeq 20)$ fiducial binaries are extremely well constrained. For example, the neutron star's mass, black hole's mass, and black hole spin are all relatively well measured, compared to the accuracy of existing measurements and hypothesized distributions of these parameters [61-63]. Higher harmonics provide relatively little additional information about these parameters.

Applied to an even simpler idealized problem-a similar source known to be directly overhead two orthogonal detectors-the effective Fisher matrix procedure of COOKL produces qualitatively similar results, notably reproducing relatively minimal impact from higher harmonics. Given the simplifications adopted, the effective Fisher matrix predictions inevitably disagree quantitatively with our detailed Monte Carlo calculations, particularly regarding multidimensional correlations. We nonetheless expect the effective Fisher matrix to correctly identify scales and trends in parameter estimation; moreover, being amenable to analysis, this simple construct allows us to develop and validate simple interpretations for why some parameters can be measured as well as they are. As a concrete example, we can explain Fig. 4.

The time-dependent orbital phase depends on the black hole spin, principally through the "aligned component" $\hat{L} \cdot \vec{S}_{1}$ [28]. As discussed in COOKL and OFOCKL, the aligned component cannot be easily distinguished from the mass ratio in the absence of precession. Spin-orbit precession breaks this degeneracy, allowing significantly tighter constraints on the mass ratio of our precessing binary. In our particular example, comparing Fig. 3 in OFOCKL to our Fig. 4, we can measure $\eta$ and hence the smaller mass roughly three times more accurately at the same signal amplitude. As seen in the bottom panel of Fig. 4, both the spin-orbit misalignment $\hat{L} \cdot \hat{S}_{1}$ and spin magnitude $\chi_{1}$ remain individually poorly constrained. As a concrete example, our ability to measure $\chi_{1}$ for this precessing binary is comparable to the accuracy possible for a similar nonprecessing binary (OFOCKL).

One correlated combination of $\hat{L} \cdot \hat{S}_{1}$ and $\chi_{1}$ is well constrained: the combination that enters into the precession 

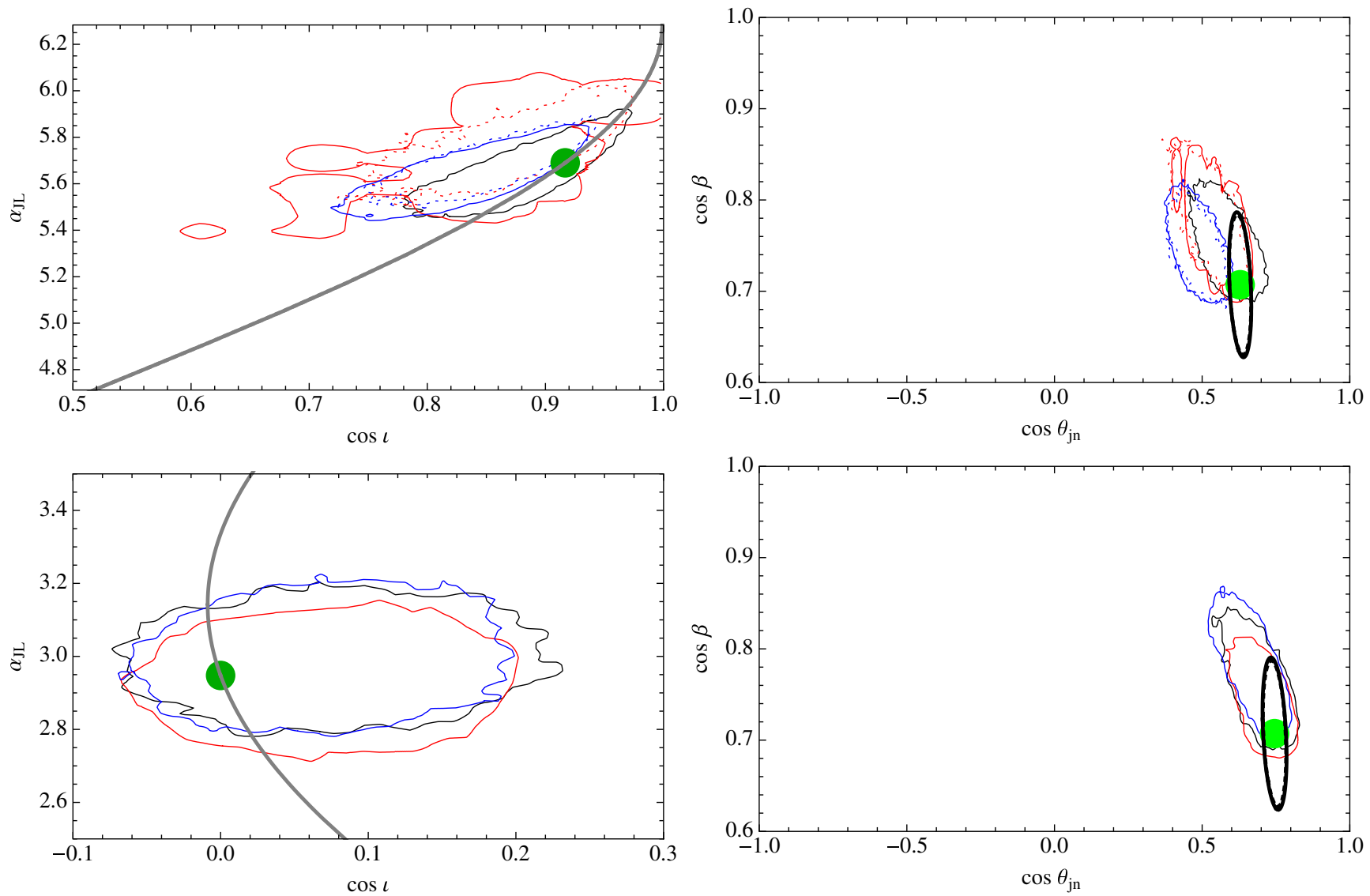

FIG. 5 (color online). Source geometry: angular momenta (C, A). For case C (top panels) and case A (bottom panels), the posterior for the precession cone (path of the angular momentum direction), expressed using the precession cone representation. This figure demonstrates that both the path $\left(\theta_{J N}, \beta_{J L}\right)$ and instantaneous orientation $\left(\alpha_{J L}, l\right)$ of the orbital angular momentum can be well determined. As in Fig. 4, colors indicate different noise realizations, solid and dotted lines indicate the neglect or use of higher harmonics, the green point shows the actual value, and the solid gray path shows the trajectory of $L$ over one precession cycle. Left panels: The precession angle $\alpha_{J L}$ of $L$ around $J$. For comparison, the green points show the simulated values; when present, the solid blue path shows variables covered in one precession cycle. Roughly speaking, the precession phase can be measured with relative accuracy tens of percent at this signal amplitude $\rho$. Right panels: Illustration that both the opening angle $\beta_{J L}$ of the precession cone and the angle $\theta_{J N}$ between the line of sight and $\hat{J}$ can be measured accurately.

rate. In Fig. 4 we show contours of constant precession cone opening angle $\left(\beta_{J L}\right)$ and constant precession rate [LO Eqs. (7)-(8)]:

$$
\Omega_{p}=\frac{|J|}{2 r^{3}}=\eta\left(2+\frac{3 m_{2}}{2 m_{1}}\right) v^{5} \sqrt{1+2 \kappa \gamma+\gamma^{2}}
$$

When evaluating these expressions, we estimate $\gamma \simeq 1.85 \chi_{1}$ [Eq. (9)], so the contours shown correspond to $\cos \beta_{J L}=0.65,0.7,0.75$ and $\sqrt{1+2 \kappa \gamma+\gamma^{2}}=2.2,2.4$, 2.6. As expected, the presence of several precession cycles allows us to relatively tightly constrain the precession rate. Future gravitational wave detectors, being sensitive to longer signals and hence more precession cycles, can be expected to even more tightly constrain this combination. By contrast, as described below, the precession geometry
$\beta_{J L}$ is relatively poorly constrained, with error independent of the number of orbital or precession cycles. ${ }^{2}$

\section{B. Geometry}

As expected analytically and demonstrated by Fig. 5, precession-induced modulations encode the orientation of the various angular momenta relative to the line of sight. For our loud fiducial signal, the individual spin components can be well constrained. Equivalently, because our fiducial source performs many precession cycles about a wide precession cone and because that source is viewed along a

\footnotetext{
${ }^{2}$ With relatively few precession cycles in our study, the discrepancy between these two measurement accuracies is fairly small. However, when advanced instruments with longer waveforms can probe more precession cycles, we expect this simple argument will explain dominant correlations.
} 
TABLE III. Simulations used in this work. Table of distinct simulations performed. The first set of columns indicates the simulated binary, whether higher harmonics were included, and random seed choice used to generate noise (a "-" means no noise was used; the asterisk indicates a different noise and MCMC realization). The two quantities $\rho_{\text {inj }}, \rho_{\text {rec }}$ provide the injected and best-fit total signal amplitude in the network [Eqs. (19) and (22) in OFOCKL]. The latter quantity depends on the noise realization of the network. The columns for $\ln Z$ and $V / V_{\text {prior }}$ provide the evidence [Eq. (15) in OFOCKL] and volume fraction [Eq. (17) of OFOCKL]; the evidence, volume fraction, and signal amplitude are related by $\rho_{\text {rec }}^{2} / 2=\ln Z /\left(V / V_{\text {prior }}\right)$.

\begin{tabular}{lccccccr}
\hline \hline Source & Harmonics & Seed & $\rho$ & $\rho_{\text {rec }}$ & $\ln Z$ & $\ln V / V_{\text {prior }}$ & $N_{\text {eff }}$ \\
\hline A & No & - & 19.86 & 20.13 & 165. & -37.9 & 10037 \\
A & No & 1234 & 19.86 & 21.26 & 186. & -40.1 & 10042 \\
A & No & 56789 & 19.86 & 20.59 & 176. & -36.4 & 10110 \\
C & No & $-*$ & 19.12 & 19.39 & 146. & -41.6 & 101600 \\
C & No & $1234^{*}$ & 19.12 & 20.64 & 169. & -43.9 & 105941 \\
C & No & $56789^{*}$ & 19.12 & 18.83 & 138. & -37.9 & 105042 \\
C & With & $1234^{*}$ & 19.73 & 21.05 & 144. & -41.8 & 42701 \\
C & With & $56789^{*}$ & 19.73 & 19.32 & 142. & -41.9 & 9814 \\
\hline \hline
\end{tabular}

generic line of sight, we can tightly constrain the precession cone's geometry: its opening angle; its orientation relative to the line of sight; and even the precise precession phase, measured either by $\cos \imath$ or $\alpha_{J L}$. The effective Fisher matrix provides a reliable estimate of how well these parameters can be measured; see Table IV and Fig. 5.

\section{Comparison to and interpretation of analytic predictions}

COOKL presented an effective Fisher matrix for two fiducial precessing binaries, adopting a specific post-Newtonian model to evolve the orbit. Following OFOCKL, we adopt a refined post-Newtonian model, including higher-order spin terms. In the Supplemental Material [64], available online, we provide a revised effective Fisher matrix, including the contribution from these terms. Table V summarizes key features of this seven-dimensional effective Fisher matrix for case A. As noted above, the two-dimensional marginalized predictions are in good qualitative agreement. The one-dimensional marginalized predictions agree surprisingly well with our simulations (Table IV). Since the ingredients of the effective Fisher matrix are fully under our analytic control, we can directly assess what factors drive measurement accuracy in each parameter.

First and foremost, as in COOKL, this effective Fisher matrix has a hierarchy of scales and eigenvalues, with decreasing measurement error: $\mathcal{M}_{c}, \eta, \chi, \ldots$ Unlike nonprecessing binaries, this hierarchy does not clearly split between well-constrained intrinsic parameters $\left(\mathcal{M}_{c}, \eta, \chi_{1}\right)$ and poorly constrained geometric parameters (everything else); for example, as seen in Table V, the eigenvalues of the Fisher matrix span a continuous range of scales.

The scales in the Fisher matrix are intimately tied to time scales and angular scales in the outgoing signal. The largest eigenvalues of the Fisher matrix are set by the shortest time scales: the orbital time scale, and changes to the orbital phase versus time. These scales control measurement of $\mathcal{M}_{c}, \eta, L \cdot a$ and set the reference event time and phase. Qualitatively speaking, we measure these parameters well

TABLE IV. One-dimensional parameter errors. Measurement accuracy $\sigma_{x}$ for $x$, one of several intrinsic $\left(\mathcal{M}_{c}, \eta, \chi_{1}\right)$, extrinsic $\left(\psi_{ \pm}, t\right.$, RA, DEC), and precession-geometry $\left(\alpha_{J L}, \beta_{J L}, \theta_{J N}\right)$ parameters. The extrinsic parameters are the event time $t$; the sky position measured in RA and DEC; and the sky area $A$, estimated using the $2 \times 2$ covariance matrix $\Sigma_{a b}$ on the sky via $\pi|\Sigma|$. The precession cone parameters are as described in Fig. 1: the precession phase $\alpha_{J L}$ at the reference frequency, the precession cone opening angle $\beta_{J L}$, and the viewing angle $\theta_{J N}$.

\begin{tabular}{lccccccccccccccc}
\hline \hline Source & Harmonics & Seed & $\rho$ & $\hat{\rho}$ & $\begin{array}{c}\sigma_{\mathcal{M}_{c}} \times 10^{3} \\
\times 10^{3}\end{array}$ & $\begin{array}{c}\sigma_{\chi_{1}} \\
(\mathrm{~ms})\end{array}$ & $\begin{array}{c}\sigma_{t} \\
(\mathrm{deg})\end{array}$ & $\begin{array}{c}\sigma_{D E C} \\
(\mathrm{deg})\end{array}$ & $\begin{array}{c}\mathrm{A} \\
\left(\mathrm{deg}^{2}\right)\end{array}$ & $\sigma_{\alpha_{J L}}$ & $\sigma_{\theta_{J N}}$ & $\sigma_{\beta_{J L}}$ & $N_{\mathrm{eff}}$ \\
\hline $\mathrm{A}$ & No & - & 19.86 & 20.13 & 5.16 & 5.62 & 0.040 & 0.505 & 0.493 & 0.747 & 1.06 & 0.094 & 0.0880 & 0.0594 & 10037 \\
$\mathrm{~A}$ & No & 1234 & 19.86 & 21.26 & 5.06 & 4.33 & 0.041 & 0.491 & 0.542 & 0.733 & 1.21 & 0.100 & 0.0891 & 0.0643 & 10042 \\
$\mathrm{~A}$ & No & 56789 & 19.86 & 20.59 & 5.08 & 4.31 & 0.033 & 0.313 & 0.572 & 0.714 & 1.27 & 0.105 & 0.0807 & 0.0523 & 10110 \\
$\mathrm{C}$ & No & $-*$ & 19.12 & 19.39 & 4.81 & 4.39 & 0.032 & 0.276 & 0.464 & 0.739 & 0.967 & 0.105 & 0.0741 & 0.0484 & 101600 \\
$\mathrm{C}$ & No & $1234^{*}$ & 19.12 & 20.64 & 4.76 & 3.72 & 0.032 & 0.247 & 0.385 & 0.647 & 0.709 & 0.0937 & 0.0624 & 0.0473 & 105941 \\
$\mathrm{C}$ & No & $56789^{*}$ & 19.12 & 18.83 & 5.40 & 4.23 & 0.039 & 0.221 & 0.469 & 0.717 & 0.960 & 0.113 & 0.0784 & 0.0622 & 105042 \\
$\mathrm{C}$ & With & $1234^{*}$ & 19.73 & 21.05 & 4.80 & 3.49 & 0.030 & 0.191 & 0.309 & 0.551 & 0.474 & 0.087 & 0.058 & 0.045 & 42701 \\
$\mathrm{C}$ & With & $56789 *$ & 19.73 & 19.32 & 4.87 & 4.20 & 0.039 & 0.191 & 0.393 & 0.661 & 0.651 & 0.112 & 0.0752 & 0.0603 & 9814 \\
\hline \hline
\end{tabular}


TABLE V. Properties of precessing effective Fisher matrix. Quantities derived from the normalized effective Fisher matrix $\hat{\Gamma}$, as provided in the Supplemental Material [64]: the eigenvalues $\lambda_{k}$ and one-dimensional parameter measurement accuracies $\sqrt{\hat{\Gamma}^{-1} / \rho^{2}}$ evaluated for $\rho=20$. (As we only compute Fisher matrices after marginalizing over $\psi$ or $\phi_{\text {ref }}$, we provide only seven eigenvalues and independent parameter measurement errors at a time.)

\begin{tabular}{lc}
\hline \hline Property & Value(s) \\
\hline$\lambda_{k}$ & $6412,673,87,5.5,0.76,0.27,0.004$ \\
$\sigma_{\mathcal{M}_{c}}$ & $0.0048 M_{\odot}$ \\
$\sigma_{\eta}$ & 0.0035 \\
$\sigma_{\chi_{1}}$ & 0.057 \\
$\sigma_{\beta_{J L}}$ & 0.06 \\
$\sigma_{\alpha_{J L}}$ & 0.10 \\
$\sigma_{\theta_{J N}}\left(\sigma_{\phi_{\text {ref }}}\right)$ & 0.07 \\
$\sigma_{\psi_{J}}$ & $0.09(0.78)$ \\
\hline \hline
\end{tabular}

because good matches require the orbital phase to be aligned over a wide range in time. We measure the reference waveform phase reliably because each waveform must be properly aligned. For this reason, parameters related to the orbital phase (i.e., $\mathcal{M}_{c}$ ) can be measured to order $1 / \sqrt{N_{\text {cycles }}}$, times suitable powers of $v$ to account for the post-Newtonian order at which those terms influence the orbital phase.

The next-shortest scales are precession scales: changes to the zero of the precession phase, and how the precession phase accumulates with time. Qualitatively speaking, we can measure the reference precession phase reliably because each precession cycle needs to be in phase. Due to spin-orbit precession, our fiducial $\mathrm{BH}-\mathrm{NS}$ binaries will undergo $N_{\text {cycles }} \simeq 10$ (30) amplitude and phase modulations in band, as seen by an initial (advanced) detector [BLO Eq. (9), for an angular-momentum-dominated binary, with $|L|>|S|]$

$$
\begin{aligned}
N_{P} & \simeq \int_{\pi f_{\min }}^{\pi f_{\max }} d f_{\text {orb }} \frac{d t}{d f_{\text {orb }}} \Omega_{p} \\
& =\frac{5}{96}\left(2+1.5 \frac{m_{2}}{m_{1}}\right)\left[\left(M \pi f_{\min }\right)^{-1}-\left(M \pi f_{\max }\right)^{-1}\right] \\
& \approx \frac{10\left(1+0.75 m_{2} / m_{1}\right)}{M / 10 M_{\odot}}\left(f_{\min } / 50 \mathrm{~Hz}\right)^{-1} .
\end{aligned}
$$

This estimate agrees favorably with the roughly 7 precession cycles performed by our spin-dominated $(|S|>|L|)$ binary between 30 and $500 \mathrm{~Hz}$ (Fig. 2). For this reason, parameters tied to spin-orbit precession rate $\Omega_{p}$ (i.e., $\eta$ ) will be measured to a relative accuracy $1 / \sqrt{N_{p}}$. Applied to the mass ratio, this estimate leads to the surprisingly successful estimate

$$
\sigma_{\eta} \simeq O(1) \times \frac{\eta}{\sqrt{N_{p}} \rho} \simeq O(1) \times 1.6 \times 10^{-3}
$$

i.e., roughly $1 / \sqrt{N_{P}}$ times smaller than the measurement accuracy possible without breaking the spin-mass ratio degeneracy.

While some parameters change the rate at which the orbital and precession phases accumulate, other reference phases simply fix the geometry. For example, a shift in the precession phase at some reference frequency [i.e., $\alpha(f=100 \mathrm{~Hz})]$ leads to a correlated shift in the precession and hence gravitational wave phase in each precession cycle. In other words, like our ability to measure the orbital phase at some time, our ability to measure the reference precession phase is essentially independent of the number of orbital or precession cycles, solely reflecting geometric factors. We expect the accuracy with which these purely geometric parameters $x$ can be determined can be estimated from first principles. To order of magnitude, we expect Fisher matrix components $\Gamma_{x x}$ comparable to $\Delta x^{2}$, where $\Delta x$ is the parameter's range. For example, the angular parameters $\left(\beta_{J L}, \theta_{J N}, \alpha, \phi\right)$ should be measured to within

$$
\sigma_{\text {angle }} \simeq \frac{(2 \pi)}{\sqrt{12} \rho} \simeq 0.09 \mathrm{rad}
$$

where the factor $\sqrt{12}$ is the standard deviation of a uniform distribution over $[0,1]$. This simple order-of-magnitude estimate compares favorably to the Fisher matrix results shown in Table $\mathrm{V}$ and to our full numerical simulations (Fig. 5 and Table IV). This naive estimate ignores all dependence on precession geometry; in general, all geometric factors are tied directly to the magnitude of precession-induced modulations, which grow increasingly significant for larger misalignment, roughly in proportion to $\cos \beta_{J L}$. This estimate for how well geometric angles can be measured should break down for nearly end-over-end precession $\left(\beta_{J L} \rightarrow \pi / 2\right)$. Nearly end-over-end precession requires extreme fine-tuning, is associated with transitional precession, and is correlated with rapid change in $\beta_{J L}$ [BLO]. We anticipate that a different set of approximations will be required to address this limit.

\section{Relative role of higher harmonics}

To this point, both our analytic and numerical calculations suggest higher harmonics provide relatively little additional information about intrinsic and extrinsic parameters. That said, as illustrated by Fig. 6, higher harmonics do break a discrete degeneracy, determining the orientation of $\hat{L}$ on the plane of the sky at $f=100 \mathrm{~Hz}$ up to a rotation by $\pi$.

OFOCKL used the evidence to demonstrate conclusively that higher harmonics had no additional impact, beyond improving knowledge of one parameter. Given expected systematic uncertainties in the evidence, at the present time 

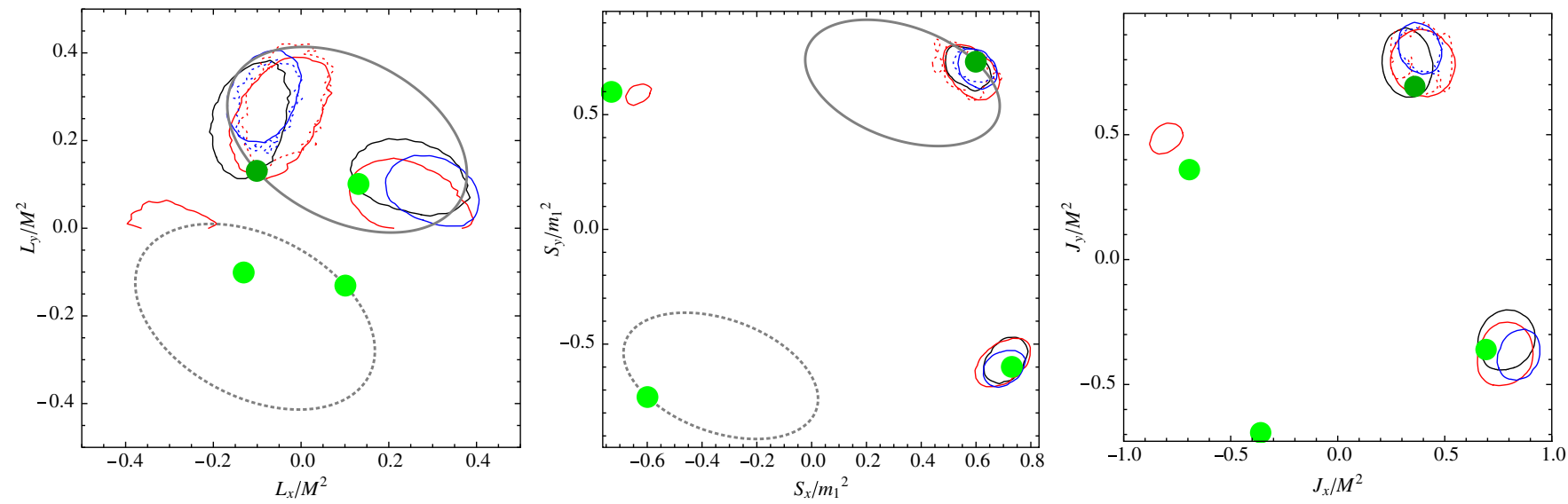

FIG. 6 (color online). Angular momentum direction on the sky $(\mathrm{C})$. Projection of the orbital angular momentum direction $(\hat{L})$ on the plane of the sky at $f=100 \mathrm{~Hz}$; compare to Fig. 2. This figure demonstrates that the individual angular momenta can be well constrained to two discrete regions; that higher harmonics allow us to distinguish between the two alternatives; and that the precession cone is well determined, at the accuracy level expected from the number of precession cycles. As in Fig. 4, colors indicate different noise realizations, solid and dotted lines indicate the neglect or use of higher harmonics, and the green point shows the expected solution.

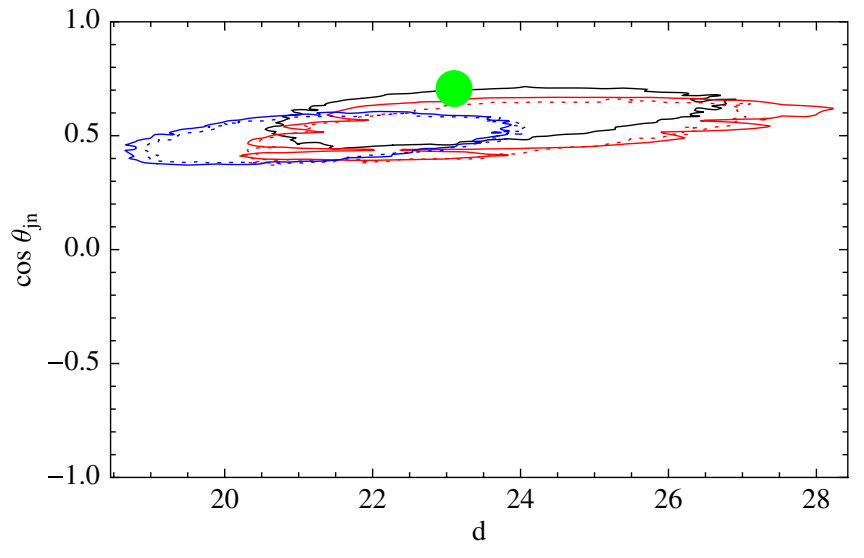

FIG. 7 (color online). Distance and inclination degeneracy broken (C). Posterior probability contours in distance and inclination.

we do not feel we can make as robust and global a statement. That said, all of our one- and two-dimensional marginalized posteriors support the same conclusion: higher harmonics provide little new information, aside from breaking one global degeneracy.

\section{E. Timing, sky location, and distance}

As seen in Fig. 7, precessing binaries do not have the strong source orientation versus distance degeneracy that plagues nonprecessing binaries: because they emit distinctively different multiharmonic signals in each direction, both the distance and emission direction can be tightly constrained.

Conversely, the sky location of precessing binaries can be determined to little better than the sky location of a nonprecessing binary with comparable signal amplitude; compare, for example, Table IV and Fig. 5 against the corresponding figures in OFOCKL.

Finally, the event time can be marginally better determined for a precessing than for a nonprecessing binary. This accuracy may be of interest for multimessenger observations of gamma ray bursts.

\section{F. Advanced versus initial instruments}

All the discussion above assumed first-generation instrumental sensitivity. For comparison and to further validate our estimates, we have also done one calculation using the expected sensitivity of second-generation instruments $[65,66]$. In this calculation, the source (event C) has been

TABLE VI. Parameter estimation with initial and advanced instruments. Like Table IV, measurement accuracy $\sigma_{x}$ for several intrinsic and extrinsic parameters. The first row provides results for initial-scale instruments, duplicating an entry in Table IV. The second row provides results for advanced detectors, operating at design sensitivity. At fixed signal amplitude, most geometric quantities can be measured to fixed accuracy, independent of detector sensitivity. Quantities impacting the orbital phase versus time (mass, mass ratio, and spin) are more accurately measured with advanced instruments, with their access to lower frequencies and hence more cycles.

\begin{tabular}{|c|c|c|c|c|c|c|c|c|c|c|c|c|c|c|c|c|}
\hline Source & Instrument & Harmonics & Noise & $\rho$ & $\hat{\rho}$ & $\begin{array}{l}\sigma_{\mathcal{M}_{c}} \\
\times 10^{3}\end{array}$ & $\begin{array}{c}\sigma_{\eta} \\
\times 10^{3}\end{array}$ & $\sigma_{\chi_{1}}$ & $\begin{array}{c}\sigma_{t} \\
(\mathrm{~ms})\end{array}$ & $\begin{array}{c}\sigma_{R A} \\
(\mathrm{deg})\end{array}$ & $\begin{array}{l}\sigma_{D E C} \\
(\mathrm{deg})\end{array}$ & $\begin{array}{c}\mathrm{A} \\
\left(\operatorname{deg}^{2}\right)\end{array}$ & $\sigma_{\alpha_{J L}}$ & $\sigma_{\theta_{J N}}$ & $\sigma_{\beta_{J L}}$ & $N_{\text {eff }}$ \\
\hline $\mathrm{C}$ & & & No & 19.12 & 19.39 & 4.81 & 4.39 & 0.032 & 0.276 & 0.464 & 0.739 & 0.967 & 0.105 & 0.0741 & 0.0484 & 101600 \\
\hline $\mathrm{C}$ & Advanced & No & Yes & 19.54 & 19.57 & 3.66 & 2.31 & 0.029 & 0.228 & 0.421 & 0.704 & 0.791 & 0.105 & 0.0699 & 0.0415 & 4004 \\
\hline
\end{tabular}




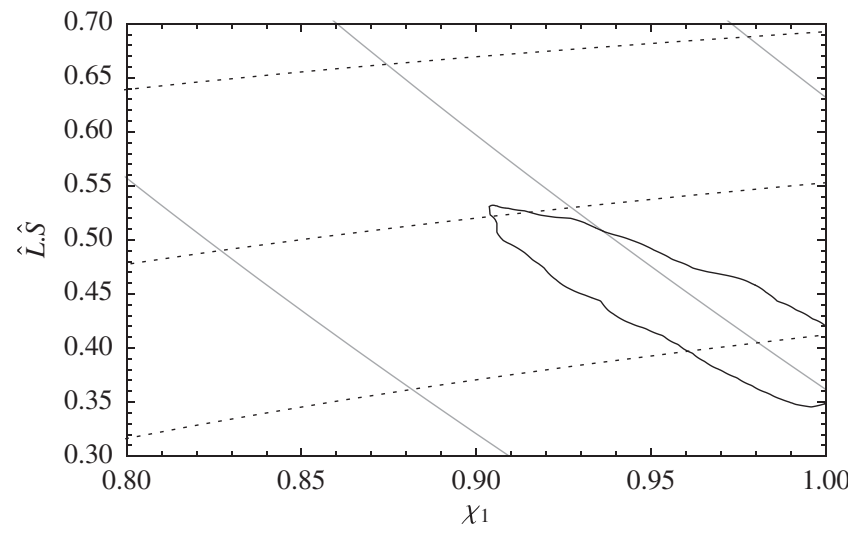

FIG. 8. Estimating astrophysical parameters with advanced detectors. Like the bottom panel of Fig. 4, but using advanced instruments; see Table VI.

placed at a larger distance $(d=298.7 \mathrm{Mpc})$ to produce the same network signal-to-noise ratio (SNR). Also, unlike the analysis above, we have for simplicity assumed the smaller compact object has no spin.

Table VI shows the resulting one-dimensional measurement accuracies, compared against a concrete simulation. All results agree with the expected scalings, as described previously. First and foremost, all geometric quantities (RA, DEC, $\left.\alpha_{J L}, \beta_{J L}, \theta_{J N}\right)$ and time can be measured to the same accuracy as in initial instruments, at fixed SNR. Second, quantities that influence the orbital decay-chirp mass, mass ratio, and spin-are all measured more precisely, because more gravitational wave cycles contribute to detection with advanced instruments. Finally, as illustrated by Fig. 8, quantities that reflect precession-induced modulation - the precession rate $\Omega_{p}$ and precession cone angle $\beta_{J L}$ misalignment - are at best measured marginally more accurately, reflecting the relatively small increase in the number of observationally accessible precession cycles for advanced detectors [Eqs. (16) and (17)]. As shown by the bottom panel of Fig. 4, this small increase in sensitivity is comparable to the typical effect of different noise realizations.

\section{CONCLUSIONS}

In this work we performed detailed parameter estimation for two selected BH-NS binaries, explained several features in terms of the binary's kinematics and geometry, and compared our results against analytic predictions using the methods of [18,27]. First, despite adopting a relatively lowsensitivity initial-detector network for consistency with prior work, we find by example that parameter estimation of precessing binaries can draw astrophysically interesting conclusions. Since our study adopted relatively bandlimited initial detector noise spectra, we expect advanced interferometers $[67,68]$ will perform at least as well (if not better) at fixed SNR. For our fiducial binaries, the mass parameters are constrained well enough to definitively say if it is a BH-NS binary (as opposed to BH-BH); the mass parameters are constrained better than similar nonprecessing binaries; and several parameters related to the spin and orientation of the binary can be measured with reasonable accuracy. Second and more importantly, we were able to explain our results qualitatively and often quantitatively using far simpler, often analytic calculations. Building on prior work by BLO, LO, and others [47], we argued precession introduced distinctive amplitude, phase, and polarization modulations on a precession time scale, effectively providing another information channel independent from the usual inspiral-scale channel found in nonprecessing binaries. Though our study targeted only two specific configurations, we anticipate many of our arguments explaining the measurement accuracy of various parameters can be extrapolated to other binary configurations and advanced detectors. The effective Fisher matrix approach of COOKL and OFOCKL provides a computationally efficient means to undertake such extrapolations. Third and finally, we demonstrated that for this mass range and orientation, higher harmonics have minimal local but significant global impact. For our systems, we found higher harmonics broke a degeneracy in the orientation of $\hat{L}$ at our reference frequency $(100 \mathrm{~Hz})$, but otherwise had negligible impact on the estimation of any other parameters.

Due to the relatively limited calculations of spin effects in post-Newtonian theory, all inferences regarding black hole spin necessarily come with significant systematic limitations. For example, Nitz et al. [69] imply that poorly constrained spin-dependent contributions to the orbital phase versus time could significantly impact parameter estimation of nonprecessing black hole-neutron star binaries. Fortunately, the leading-order precession equations and physics are relatively well determined. For example, the amplitude of precession-induced modulations is set by the relative magnitude and misalignment of $\vec{L}$ and $\vec{S}_{1}$. In our opinion, the leading-order symmetry-breaking effects of precession are less likely to be susceptible to systematic error than high-order corrections to the orbital phase. Significantly more study would be needed to validate this hypothesis.

Robust though these correlations may be, the quantities that gravitational wave measurements naturally provide (chirp mass, precession rate, geometry) rarely correspond to astrophysical questions. We have demonstrated by example that measurements of relatively strong gravitational wave signals can distinguish individual component masses and spins to astrophysically interesting accuracy (Fig. 4). Given the accuracy and number of measurements gravitational waves will provide, compared to existing astrophysical experience [70-74], these measurements should transform our understanding of the lives and deaths of massive stars. Ignoring correlations, gravitational wave measurements seem to only relatively weakly constrain 
spin-orbit misalignment (Fig. 4), a proxy for several processes including supernova kicks and stellar dynamics. That said, gravitational wave measurements should strongly constrain the precession rate, a known expression of spins, masses, and spin-orbit misalignment. Formation models which make nontrivial predictions about both spin magnitude and misalignment might therefore be put to a strong test with gravitational wave measurements.

\section{ACKNOWLEDGMENTS}

This material is based upon work supported by the National Science Foundation under Grants No. PHY0970074, No. PHY-0923409, No. PHY-1126812, and No. PHY-1307429. R. O.S. acknowledges support from the UWM Research Growth Initiative. B. F. is supported by an NSF fellowship DGE-0824162. V. R. was supported by a Richard Chase Tolman fellowship at the California Institute of Technology. H. S. C., C. K. and C. H. L. are supported in part by the National Research Foundation Grant funded by the Korean Government (No. NRF-2011220-C00029) and the Global Science Experimental Data Hub Center (GSDC) at KISTI. H. S. C. and C. H. L. are supported in part by the BAERI Nuclear R\&D program (No. M20808740002). This work uses computing resources both at KISTI and CIERA, the latter funded by NSF Grant. No. PHY-1126812.

\section{APPENDIX: MORE PROPERTIES OF THE EFFECTIVE FISHER MATRIX}

\section{Separation of scales and mutual information}

On physical grounds, we expect the time scales and modulations produced by precession to separate, allowing roughly independent measurements of orbital- and precession-rate-related parameters (i.e., $\mathcal{M}_{c}, \eta, a, \beta_{J L}$ ) and purely geometric parameters $(\alpha, \phi, \theta)$. To assess this hypothesis quantitatively, we evaluate the mutual information between the two subspaces. For a Gaussian distribution described by a covariance matrix $\Gamma$, the mutual information between two subspaces $A, B$ is [OFOCKL Eq. (31)]

$$
I(A, B)=-\frac{1}{2} \ln \frac{|\Gamma|}{\left|\Gamma_{A}\right|\left|\Gamma_{B}\right|} .
$$

Table VII shows that after marginalizing out orbital phase $\left(\phi_{\text {ref }}\right)$, the mutual information between orbital-phaserelated parameters $\left(\mathcal{M}_{c}, \eta, a, \beta_{J L}\right)$ and geometric parameters is small but nonzero (0.31): the two subspaces are
TABLE VII. Separation of variables in the precessing effective Fisher matrix. For several different subspaces $A, B$ and marginalized parameters $C$, the mutual information $I(A, B \mid C)$.

\begin{tabular}{lc}
\hline \hline Type of joint information & Value \\
\hline$I\left(\left\{\mathcal{M}_{c}, \eta, \chi_{1}, \beta_{J L}\right\},\left\{\theta_{J N}, \alpha_{J L}\right\} \mid \phi_{\text {ref }}\right)$ & 0.31 \\
$I\left(\left\{\theta_{J N}\right\},\left\{\alpha_{J L}\right\} \mid\left\{\mathcal{M}_{c}, \eta, \chi_{1}, \beta_{J L}, \phi_{\text {ref }}\right\}\right)$ & 0.01 \\
$I\left(\left\{\mathcal{M}_{c}\right\},\left\{\eta, \chi_{1}, \beta_{J L}\right\} \mid\left\{\theta_{J N}, \alpha_{L N}, \phi\right\}\right)$ & 1.92 \\
\hline \hline
\end{tabular}

weakly correlated. By comparison, the mutual information $I(a, c \mid B)$ between two intrinsic parameters $a, c$ in $A=$ $\left\{\mathcal{M}_{c}, \eta, \chi_{1}\right\}$ is large. Finally, after marginalizing out all other parameters, the mutual information between $\alpha_{J L}$ and $\left(\theta_{J N}\right)$ is small, as expected given the different forms in which these quantities enter into the outgoing gravitational wave signal.

\section{Regularizing calculations with a prior}

Due to the wide range of eigenvalues and poor condition number, all Fisher matrices are prone to numerical instability in high dimension. Additionally, due to physical neardegeneracies, the error ellipsoid derived from the Fisher matrix alone may extend significantly outside the prior range; see, e.g., examples in [75].

Following convention, to ensure our results are stable to physical limitations, we derive parameter measurements' accuracies $\Sigma=\Gamma^{-1} / \rho^{2}$ by combining the signal amplitude $\rho$, the normalized effective Fisher matrix $\hat{\Gamma}^{\text {eff }}$ provided above, and a prior $\Gamma^{\text {prior: }}$

$$
\Gamma_{\lambda}^{\mathrm{eff}} \equiv \rho^{2} \hat{\Gamma}^{\mathrm{eff}}+\lambda \Gamma^{\mathrm{prior}}
$$

$$
\begin{aligned}
\Gamma^{\text {prior }}= & e_{\eta} \otimes e_{\eta}+\frac{e_{a} \otimes e_{a}}{20} \\
& +\frac{1}{(2 \pi)^{2}}\left[e_{\beta} \otimes e_{\beta}+e_{\theta} \otimes e_{\theta}+e_{\alpha} \otimes e_{\alpha}+e_{\phi} \otimes e_{\phi}\right] .
\end{aligned}
$$

As expected given the eigenvalues and signal amplitude, this prior has no significant impact on our calculations. In particular, the eigenvalues and parameter measurement accuracies reported in the text are unchanged if this weak prior is included. 
[1] B. P. Abbott et al. (LIGO Scientific Collaboration), Rep. Prog. Phys. 72, 076901 (2009).

[2] T. Accadia et al., JINST 7, P03012 (2012).

[3] A. Buonanno, Y. Chen, and M. Vallisneri, Phys. Rev. D 67, 104025 (2003).

[4] A. Buonanno, Y. Chen, Y. Pan, and M. Vallisneri, Phys. Rev. D 70, 104003 (2004).

[5] T. Damour, A. Gopakumar, and B. R. Iyer, Phys. Rev. D 70, 064028 (2004).

[6] Y. Pan, A. Buonanno, Y. Chen, and M. Vallisneri, Phys. Rev. D 69, 104017 (2004).

[7] C. Königsdörffer and A. Gopakumar, Phys. Rev. D 71, 024039 (2005).

[8] A. Buonanno, Y. Chen, Y. Pan, H. Tagoshi, and M. Vallisneri, Phys. Rev. D 72, 084027 (2005).

[9] C. Königsdörffer and A. Gopakumar, Phys. Rev. D 73, 124012 (2006).

[10] M. Tessmer and A. Gopakumar, Mon. Not. R. Astron. Soc. 374, 721 (2007).

[11] M. Hannam, S. Husa, B. Brügmann, and A. Gopakumar, Phys. Rev. D 78, 104007 (2008).

[12] I. Hinder, F. Herrmann, P. Laguna, and D. Shoemaker, Phys. Rev. D 82, 4033 (2010).

[13] K. G. Arun, A. Buonanno, G. Faye, and E. Ochsner, Phys. Rev. D 79, 104023 (2009).

[14] A. Buonanno, B. R. Iyer, E. Ochsner, Y. Pan, and B. S. Sathyaprakash, Phys. Rev. D 80, 084043 (2009).

[15] D. A. Brown, A. Lundgren, and R. O'Shaughnessy, Phys. Rev. D 86, 064020 (2012).

[16] A. Lundgren and R. O'Shaughnessy, Phys. Rev. D 89, 044021 (2014).

[17] T. A. Apostolatos, C. Cutler, G. J. Sussman, and K. S. Thorne, Phys. Rev. D 49, 6274 (1994).

[18] H. Cho, E. Ochsner, R. O'Shaughnessy, C. Kim, and C. Lee, Phys. Rev. D 87, 024004 (2013).

[19] J. Aasi et al. (LIGO Scientific Collaboration and Virgo Collaboration), Phys. Rev. D 88, 062001 (2013).

[20] H.-T. Janka, Mon. Not. R. Astron. Soc. 434, 1355 (2013).

[21] J. Nordhaus, T. D. Brandt, A. Burrows, and A. Almgren, Mon. Not. R. Astron. Soc. 423, 1805 (2012).

[22] D. Gerosa, M. Kesden, E. Berti, R. O'Shaughnessy, and U. Sperhake, Phys. Rev. D 87, 104028 (2013).

[23] H. van Eerten, W. Zhang, and A. MacFadyen, Astrophys. J. 722, 235 (2010).

[24] H. J. van Eerten and A. I. MacFadyen, Astrophys. J. Lett. 733, L37 (2011).

[25] H. van Eerten and A. MacFadyen, Astrophys. J. 767, 141 (2013).

[26] N. Gehrels, E. Ramirez-Ruiz, and D. B. Fox, Annu. Rev. Astron. Astrophys. 47, 567 (2009).

[27] R. O'Shaughnessy, B. Farr, E. Ochsner, C. Cho, H. S. Kim, and C. Lee, Phys. Rev. D 89, 064048 (2014).

[28] E. Poisson and C. M. Will, Phys. Rev. D 52, 848 (1995).

[29] E. Baird, S. Fairhurst, M. Hannam, and P. Murphy, Phys. Rev. D 87, 024035 (2013).

[30] R. N. Lang and S. A. Hughes, Phys. Rev. D 74, 122001 (2006).

[31] A. Klein, P. Jetzer, and M. Sereno, Phys. Rev. D 80, 064027 (2009).
[32] R. N. Lang, S. A. Hughes, and N. J. Cornish, Phys. Rev. D 84, 022002 (2011).

[33] C. Van Den Broeck and A. S. Sengupta, Classical Quantum Gravity 24, 155 (2007).

[34] V. Raymond, Ph.D. thesis, Northwestern University, 2012, https://gwic.ligo.org/thesisprize/2012/raymond-thesis.pdf.

[35] J. Veitch, I. Mandel, B. Aylott, B. Farr, V. Raymond, C. Rodriguez, M. van der Sluys, V. Kalogera, and A. Vecchio, Phys. Rev. D 85, 104045 (2012).

[36] B. Farr et al. https://trac.ligo.caltech.edu/cbc/browser/pe/ papers/lalinference.

[37] W. Del Pozzo, J. Veitch, and A. Vecchio, Phys. Rev. D 83, 082002 (2011).

[38] N. Cornish, L. Sampson, N. Yunes, and F. Pretorius, Phys. Rev. D 84, 062003 (2011).

[39] S. Nissanke, J. Sievers, N. Dalal, and D. Holz, Astrophys. J. 739, 99 (2011).

[40] T. G. F. Li, W. Del Pozzo, S. Vitale, C. Van Den Broeck, M. Agathos, J. Veitch, K. Grover, T. Sidery, R. Sturani, and A. Vecchio, Phys. Rev. D 85, 082003 (2012).

[41] J. Veitch, I. Mandel, B. Aylott, B. Farr, V. Raymond, C. Rodriguez, M. van der Sluys, V. Kalogera, and A. Vecchio, Phys. Rev. D 85, 104045 (2012).

[42] V. Raymond and W. M. Farr, arXiv:1402.0053.

[43] B. Farr and O. Oscher (to be published).

[44] E. Ochsner and R. O’Shaughnessy, Phys. Rev. D 86, 104037 (2012).

[45] D. A. Brown, I. Harry, A. Lundgren, and A. H. Nitz, Phys. Rev. D 86, 084017 (2012).

[46] A. Klein, N. Cornish, and N. Yunes, Phys. Rev. D 88, 124015 (2013).

[47] R. O'Shaughnessy, L. London, J. Healy, and D. Shoemaker, Phys. Rev. D 87, 044038 (2013).

[48] L. Pekowsky, R. O'Shaughnessy, J. Healy, and D. Shoemaker, Phys. Rev. D 88, 024040 (2013).

[49] M. Hannam, P. Schmidt, A. Bohé, L. Haegel, S. Husa, F. Ohme, G. Pratten, and M. Pürrer, arXiv:1308.3271.

[50] M. Boyle, R. Owen, and H. P. Pfeiffer, Phys. Rev. D 84, 124011 (2011).

[51] P. Schmidt, M. Hannam, and S. Husa, Phys. Rev. D 86, 104063 (2012).

[52] P. Schmidt, M. Hannam, S. Husa, and P. Ajith, Phys. Rev. D 84, 024046 (2011).

[53] J. D. Schnittman, Phys. Rev. D 70, 124020 (2004).

[54] T. Damour, P. Jaranowski, and G. Schfer, Phys. Rev. D 78, 024009 (2008).

[55] J. Steinhoff, G. Schäfer, and S. Hergt, Phys. Rev. D 77, 104018 (2008)

[56] J. Levin, S. T. McWilliams, and H. Contreras, Classical Quantum Gravity 28, 175001 (2011).

[57] A. Nagar, Phys. Rev. D 84, 084028 (2011).

[58] J. Hartung, J. Steinhoff, and G. Schfer, Ann. Phys. (Berlin) 525, 359 (2013).

[59] M. Tessmer, J. Steinhoff, and G. Schäfer, Phys. Rev. D 87, 064035 (2013).

[60] R. O'Shaughnessy, B. Vaishnav, J. Healy, Z. Meeks, and D. Shoemaker, Phys. Rev. D 84, 124002 (2011).

[61] F. Özel, D. Psaltis, R. Narayan, and A. Santos Villarreal, Astrophys. J. 757, 55 (2012). 
[62] L. Kreidberg, C. D. Bailyn, W. M. Farr, and V. Kalogera, Astrophys. J. 757, 36 (2012).

[63] M. Hannam, D. A. Brown, S. Fairhurst, C. L. Fryer, and I. W. Harry, Astrophys. J. Lett. 766, L14 (2013).

[64] See Supplemental Material at http://link.aps.org/supplemental/ 10.1103/PhysRevD.89.102005 for the effective Fisher matrix used in this work.

[65] D. Shoemaker (LIGO Scientific Collaboration), https://dcc .ligo.org/cgi-bin/DocDB/ShowDocument?docid=m060056.

[66] J. Abadie, B. P. Abbott, R. Abbott, M. Abernathy, T. Accadia, F. Acernese, C. Adams, R. Adhikari, P. Ajith, B. Allen et al., Classical Quantum Gravity 27, 173001 (2010).

[67] J. Abadie et al. (LIGO-Virgo Scientific Collaboration), Classical Quantum Gravity 27, 173001 (2010).
[68] G. M. Harry (LIGO Scientific Collaboration), Classical Quantum Gravity 27, 084006 (2010).

[69] A. H. Nitz, A. Lundgren, D. A. Brown, E. Ochsner, D. Keppel, and I. W. Harry, Phys. Rev. D 88, 124039 (2013).

[70] R. O'Shaughnessy, Phys. Rev. D 88, 084061 (2013).

[71] I. Mandel and R. O'Shaughnessy, Classical Quantum Gravity 27, 114007 (2010).

[72] F. Özel, D. Psaltis, R. Narayan, and J. E. McClintock, Astrophys. J. 725, 1918 (2010).

[73] F. Özel, D. Psaltis, R. Narayan, and A. Santos Villarreal, Astrophys. J. 757, 55 (2012).

[74] B. Kiziltan, A. Kottas, M. De Yoreo, and S. E. Thorsett, Astrophys. J. 778, 66 (2013).

[75] M. Wade, J. D. E. Creighton, E. Ochsner, and A. B. Nielsen, Phys. Rev. D 88, 083002 (2013). 